\title{
Chitosan-reinforced alginate microspheres obtained through the emulsification/internal gelation technique
}

\author{
António J. Ribeiro ${ }^{\mathrm{a}, *}$, Catarina Silva ${ }^{\mathrm{b}}$, Domingos Ferreira ${ }^{\mathrm{c}}$, Francisco Veiga ${ }^{\mathrm{b}}$ \\ a Laboratório de Tecnologia Farmacêutica, Instituto Superior de Ciências da Saúde-Norte, 4585-116 Gandra, Portugal \\ ${ }^{\mathrm{b}}$ Laboratório de Tecnologia Farmacêutica, Faculdade de Farmácia, Universidade de Coimbra, Portugal \\ ${ }^{\mathrm{c}}$ Laboratório de Tecnologia Farmacêutica, Faculdade de Farmácia, Universidade do Porto, Portugal
}

Received 29 April 2004; received in revised form 11 January 2005; accepted 14 January 2005

Available online 3 March 2005

\begin{abstract}
Alginate microspheres prepared by emulsification/internal gelation were chosen as carriers for a model protein, hemoglobin (Hb). Reinforced chitosan-coated microspheres were obtained by an uninterrupted method, in order to simplify the coating process, minimize protein losses during production and to avoid $\mathrm{Hb}$ escape under acidic conditions. Microspheres recovery was evaluated as well as its morphology by determination of $\mathrm{Hb}$ encapsulation efficiency and microscopic observation, respectively. The formation of chitosan membrane made of it interaction with alginate was assessed by DSC (differential scanning calorimetry) and FT-IR (Fourier-transform infrared spectrometry) studies. Spherical uncoated microspheres with a mean diameter of $20 \mu \mathrm{m}$ and encapsulation efficiency above $89 \%$ were obtained. Coated microspheres provided similar encapsulation efficiency but a higher mean diameter was obtained due to microspheres clumping during the coating step. Protein loss occurred mainly during emulsification rather than recovery. FT-IR and DSC together indicated electrostatic interactions between alginate carboxylate and chitosan ammonium groups as the main forces for complex formation.

$\mathrm{Hb}$ release from microspheres showed a $\mathrm{pH}$-dependent profile and was affected by chitosan coating. Under simulated gastric conditions, a total $\mathrm{Hb}$ burst release from uncoated microspheres was decreased with one-stage and two-stage chitosan coatings (68\% and $28 \%$, respectively). At $\mathrm{pH}$ 6.8, the $\mathrm{Hb}$ release from coated microspheres was fast but incomplete. These results suggest an optimization of the coating method to protect $\mathrm{Hb}$ under acidic conditions and to permit a complete but sustained release of $\mathrm{Hb}$.
\end{abstract}

(c) 2005 Elsevier B.V. All rights reserved.

Keywords: Alginate; Chitosan; Internal gelation; Microspheres; Oral protein delivery

\section{Introduction}

Protein drugs are mostly delivered by parenteral administration. To minimize the health hazard by repeated injection of these short-acting drugs, there is an urgent need to develop a non-parenteral route of administration as well as to develop formulations with controlled-delivery features.

The applicability of microspheres as oral protein delivery systems is based not only on protection from gastrointestinal (GI) conditions, but also on the fact that the release profile of encapsulant can be adjusted by polymer degradation.

\footnotetext{
* Corresponding author. Tel.: +351 224157185 .

E-mail address: a_j_ribeiro@sapo.pt (A.J. Ribeiro).
}

Alginate and chitosan, naturally occurring biopolymers, have shown potential for use as scaffolds in tissue-engineered medical products (Machluf et al., 2000; Pariente et al., 2001), as an encapsulating matrix for immobilization of living cells (Stevens et al., 2004), and as drug delivery systems (Chandy et al., 2002; Onal and Zihnioglu, 2002).

To date, the production of alginate gel microspheres has been accomplished mainly using external gelation; alginate is extruded drop-wise through a needle into a solution of divalent cations, which induces cross-linking of the guluronic residues of the alginate polymer (Kierstan and Bucke, 1977). Although this procedure has been used to encapsulate a wide variety of proteins such as albumin (Coppi et al., 2002; Wang and $\mathrm{He}, 2002$ ), growth factors (Chinen et al., 2003; Ko et al., 
1995), DNA (Quong and Neufeld, 1998) and $\mathrm{Hb}$ (Huguet et al., 1994), industrial scale-up is complicated, resulting in an awkward production system (Poncelet et al., 1992a). Furthermore, it is difficult to obtain lower size microparticles, which are preferred either to stabilize the fragile structure of the proteins to keep it biologically active and to control delivery kinetics. Moreover, physical hydrogels are not homogeneous, since clusters of ionically associated domains can create inhomogeneties (Hoffman, 2001). The production of alginate microspheres via internal gelation offers a scalable method of encapsulation of cells (Larisch et al., 1994), DNA (Quong et al., 1998) and drugs (Ribeiro et al., 1999) and is based on the release of calcium ion from an acid soluble calcium salt, in emulsified sodium alginate solution. This is achieved by acidification with an oil-soluble acid, which partitions to the dispersed aqueous alginate phase, releasing soluble calcium, initiating gelation.

One of the limitations of this technique lies in the ability to encapsulate proteins with high encapsulation efficiency. It has been reported that encapsulant loss is due to the homogeneous nature of the alginate microspheres (Quong et al., 1998); protein escape from the internal to the external phase during emulsification (Vandenberg and Noue, 2001) and microsphere recovery steps. Moreover, alginate microspheres instability in a phosphate buffer above $\mathrm{pH} 5$ is a drawback in the design of controlled release dosage forms (Chan and Heng, 2002).

Chitosan, a polysaccharide derived from chitin by alkaline deacetylation, has been used to reinforce alginate microspheres (Murata et al., 1993) based on the electrostatic interaction between carboxylate alginate groups and ammonium chitosan groups (Haas et al., 1996). Chitosan-alginate complex erode slowly in phosphate buffer, and this behavior leads to suppression of the initial release of drugs occurring for uncoated microspheres (Liu et al., 1997; Murata et al., 1993). Usually these microspheres are produced by a twostage method, where preformed alginate microspheres are recovered and subsequently coated with chitosan. Chitosancoating of low-sizing alginate microspheres is an awkward technique and the possibility of doing it during procedure by a simple method can be a valid alternative. Furthermore, it would contribute to retain encapsulant during emulsification, decrease its loss during microspheres recovery, and may provide a sustained release effect.

The purpose of the present work was to encapsulate proteins into alginate microspheres by emulsification/internal gelation with high encapsulation efficiency. Microspheres formulation was realized by using either $\mathrm{pH}$ conditions and applying a chitosan-coat to alginate microspheres in order to favour protein encapsulation. In order to simplify coating process, chitosan membrane was applied by a scalable one-stage method and compared to the classic two-stage process. Infrared (IR) spectra and differential scanning calorimetry (DSC) were used to assess alginate-chitosan interactions, the microspheres structure, and to determine whether this uninterrupted coating method allows cross-linking when com- pared to the classical coating process. Hemoglobin, a coloredprotein, was selected as protein model to follow microspheres recovery from the bi-phasic system and encapsulant release under simulated GI intestinal conditions.

\section{Materials and methods}

\subsection{Materials}

Alginate SG 300 was supplied by KWR Biosystems (Paris, France). This alginate exhibits an average molecular weight of $694 \mathrm{KDa}$ and a guluronic content of 60\% (Quong et al., 1998). Paraffin oil and glacial acetic acid were supplied by Vaz Pereira (Lisboa, Portugal). Micronized $\mathrm{CaCO}_{3}$ was obtained from Omya (Orgon, France), Tween $80^{\circledR}$, Span $80^{\circledR}$, and bovine methemoglobin $(\mathrm{Hb})$ were purchased from Sigma (Madrid, Spain). Hemoglobin has a molecular weight of $64.5 \mathrm{KDa}$ and an isoelectric point of 7.1 (as provided by the supplier). Hydrochloride chitosan, having an average molecular weight of $150 \mathrm{KDa}$ and a deacetylation degree of $85 \%$ (as provided by the supplier) was purchased from Fluka (Madrid, Spain).

\subsection{Microsphere preparation}

The emulsification/internal gelation technique to form alginate microspheres has been described previously (Poncelet et al., 1992b). Sodium alginate $2.0 \%$ (w/v) was dispersed into phosphate buffer at $\mathrm{pH} 6.8$ (USP XXV) containing $\mathrm{Hb}$ at $1.5 \%(\mathrm{w} / \mathrm{v})$, by using an orbital shaker $(200 \mathrm{rpm} / 12 \mathrm{~h})$. Microcrystalline $\mathrm{CaCO}_{3}(5 \%$, w/v) was added into the alginate $(\mathrm{Ca} /$ alginate $24 \%, \mathrm{w} / \mathrm{w})$ and the mixture was dispersed into paraffin oil containing $1 \%$ of Span $80^{\circledR}$ by stirring at $400 \mathrm{rpm}$ using an IKA ${ }^{\circledR}$-Werke Eurostar mixer (IKA, Staufen, Germany) with a marine impeller. The ratio between alginate and oily phases was 30/70 (v/v). After 15 min emulsification, $20 \mathrm{ml}$ of paraffin oil containing $500 \mu \mathrm{l}$ of glacial acetic acid were added and stirring continued for $60 \mathrm{~min}$ to permit the solubilization of the calcium carbonate. Alginate gelled microspheres (UI) were first washed by adding $100 \mathrm{ml}$ of acetate buffer $\mathrm{pH} 5.5$ (USP XXV) followed by orbital agitation $(200 \mathrm{rpm} / 10 \mathrm{~min})$. Microspheres settled down after $12 \mathrm{~h}$ at $4-8^{\circ} \mathrm{C}$ and the top layer oily phase was removed by aspiration. Subsequent washes were performed by adding washing medium, orbital agitation ( $200 \mathrm{rpm} / 15 \mathrm{~min})$, microspheres sedimentation and oil separation, until no residual oil was observed by optical microscope observation.

\subsection{Preparation of chitosan-coated alginate microspheres}

Chitosan-coating of microspheres obtained by internal gelation was performed by using two different methods. In the one-stage method (C1I) an emulsion of $0.3 \%(\mathrm{w} / \mathrm{v})$ aqueous chitosan at $\mathrm{pH} 5.6$ in paraffin oil $(50 / 50, \mathrm{v} / \mathrm{v})$ was added 
to the oily dispersed gelled microspheres after calcium solubilization and stirring was allowed for $30 \mathrm{~min}$. Coated microspheres were recovered as described above. In the two-stage method (C2I), microspheres were isolated and then transferred into a $0.3 \%(\mathrm{w} / \mathrm{v})$ aqueous chitosan solution at $\mathrm{pH} 5.6$ under magnetic stirring for $30 \mathrm{~min}$.

\subsection{Microsphere morphology}

Microsphere features such as shape and existence of aggregates was examined after isolation by using an optical microscope Nikon eclipse TE 2000-U equipped with a Nikon digital camera DXM 1200 F. Scanning electron microscopy (SEM) was utilized to compare microspheres morphology, especially the surface characteristics of uncoated- and coatedmicrospheres. Samples were fixed on an SEM sample holder, coated with a thin layer of gold and then observed with a JEOL JSM-840 ${ }^{\circledR} 10 \mathrm{kv}$.

Volumetric size distribution (volume of microspheres in each diameter by class) of the microspheres was determined by laser diffractometry with a Coulter LS130 particle analyzer with the mean diameter and standard deviation calculated from the cumulative distribution curve.

\subsection{Encapsulation efficiency}

$\mathrm{Hb}$ losses during microspheres first and subsequent washes were determined by an adaptation of a cyanohemoglobin method (Suzuki, 1998). Samples taken from washing media were mixed with EDTA $0.1 \mathrm{M}-\mathrm{NaOH} 0.1 \mathrm{M}$ to promote hemoglobin hydrolysis. Alginate was precipitated by adding ethanol (50/50, v/v) and together with chitosan was separated from the $\mathrm{Hb}$ derivate-dissolved supernatant by centrifugation $(3000 \mathrm{rpm} / 1 \mathrm{~min})$. The absorbance was measured at $401 \mathrm{~nm}$ (UV-1603 UV-vis spectrophotometer, Shimadzu, Kyoto, Japan). A calibration curve was prepared for up to $0.6 \mathrm{~g} \mathrm{l}^{-1}$ of $\mathrm{Hb}$ concentration. The encapsulation efficiency of $\mathrm{Hb}$ was calculated from the difference between it total losses and the initial amount of protein.

\subsection{Differential scanning calorimetry}

DSC was used to determine a shift of the alginate endothermic peak or the appearance of exothermic peaks and consequently detect interactions between chitosan and alginate. Thermograms were obtained using a Shimadzu DSC50 system (Shimadzu, Kyoto, Japan). Sodium alginate and chitosan acetate were obtained by lyophilization of aqueous solution $0.3 \%(\mathrm{w} / \mathrm{v})$ and an aqueous acetic acid solution $0.3 \%(\mathrm{w} / \mathrm{v})$, respectively. Physical mixture was prepared by mixing $(1 / 1, w / w)$ lyophilized sodium alginate with chitosan acetate. Chitosan-alginate (CS-ALG) complexes were obtained by adding $10 \mathrm{ml}$ of chitosan solution $0.3 \%(\mathrm{w} / \mathrm{v})$ at $\mathrm{pH} 6.4$ to $10 \mathrm{ml}$ of alginate solution $0.3 \%(\mathrm{w} / \mathrm{v})$ at $\mathrm{pH} 4.5$ under agitation for $20 \mathrm{~min}$ followed by lyophilization. Blank microspheres were obtained either by external and internal gelation. External gelation of alginate was performed by extruding a $2 \%(\mathrm{w} / \mathrm{v})$ aqueous alginate into a $0.05 \mathrm{M}$ calcium chloride solution. Microspheres were isolated by filtration, washed with water and lyophilized (UEL). Chitosan-coated microspheres were obtained by transferring alginate microspheres to a $0.3 \%(\mathrm{w} / \mathrm{v})$ chitosan solution and allowed to react for $30 \mathrm{~min}$. Microspheres were isolated by filtration, washed with water and lyophilized (CEL). Microspheres obtained by internal gelation were lyophilized and used as uncoated (UIL); one stage-coated (C1IL) and two-stage coated (C2IL).

Samples were crimped in a standard aluminium pan and heated from 20 to $350^{\circ} \mathrm{C}$ at a heating rate of $10^{\circ} \mathrm{C} / \mathrm{min}$ under constant purging of nitrogen at $20 \mathrm{ml} / \mathrm{min}$.

\subsection{Measurements of Fourier transform infrared spectrometry}

Infrared (IR) spectra of alginate, chitosan, and alginate-chitosan complex were recorded with a JASCO FT/IR-420 spectrophotometer (Miracle, PIKE ${ }^{\circledR}$ Technologies) spectrometer using the attenuated total reflection (ATR) method. Samples were scanned from 600 to $4000 \mathrm{~cm}^{-1}$ at a resolution of $4 \mathrm{~cm}^{-1}$. Samples were prepared as described for DSC analysis.

\subsection{In vitro release studies}

Hydrochloric acid ( $\mathrm{HCl}$ ) buffer $\mathrm{pH} 1.2$ (USP XXV) and phosphate buffer (PB) pH 6.8 (USP XXV) were used to simulate GI intestinal conditions. An amount of microspheres equivalent to $10 \mathrm{mg}$ of $\mathrm{Hb}$ was placed in glass vials containing $50 \mathrm{ml}$ of $\mathrm{HCl}$, under magnetic stirring at $100 \mathrm{rpm}$. After $2 \mathrm{~h}$, microspheres were transferred into PB pH 6.8. Samples were withdrawn at different time intervals and spectrophotometrically assayed for the $\mathrm{Hb}$ concentration as mentioned previously. $\mathrm{Hb}$ released was calculated accordingly to the following equation:

$\mathrm{Hb}$ release $=\frac{M_{t}}{M_{t_{0}}} \times 100$

where $M_{t}$ is the amount of $\mathrm{Hb}$ at time $t$ and $M_{t_{0}}$ the amount of $\mathrm{Hb}$ in the microspheres at time $t=0$. All experiments were done at least in triplicate.

\section{Results}

$\mathrm{Hb}$-dissolved alginate aqueous phase was emulsified into paraffin oil and the ionized alginate carboxylic groups were cross-linked by an internal source of calcium resulting in oildispersed gelled alginate microspheres.

A high encapsulation efficiency, above $89 \%$, was obtained for both uncoated and coated microspheres (Table 1) and $\mathrm{Hb}$ losses occurred mainly during first wash of microspheres.

The morphology of microspheres was determined in terms of final shape and granulometry. Optical micrographs (Fig. 1) 
Table 1

$\mathrm{Hb}$ losses during first and subsequent washes of microspheres, required to isolate microspheres

\begin{tabular}{|c|c|c|c|c|}
\hline \multirow[t]{2}{*}{ Microspheres } & \multicolumn{2}{|l|}{ Source of loss } & \multirow[t]{2}{*}{ Total loss $(\%)$} & \multirow[t]{2}{*}{ Hb encapsulation efficiency $(\%)$} \\
\hline & First wash (\%) & Subsequent washes (\%) & & \\
\hline UI & $7.6 \pm 2.7$ & $1.5 \pm 0.4$ & $9.1 \pm 2.7$ & $90.9 \pm 2.7$ \\
\hline C1I & $10.2 \pm 2.4$ & $2.0 \pm 2.3$ & $10.6 \pm 4.4$ & $89.4 \pm 4.4$ \\
\hline $\mathrm{C} 2 \mathrm{I}$ & $7.6 \pm 2.7$ & $1.5 \pm 0.4$ & $9.1 \pm 2.7$ & $90.9 \pm 2.7^{\mathrm{a}}$ \\
\hline
\end{tabular}

${ }^{\text {a }} \mathrm{No} \mathrm{Hb}$ was found in the chitosan solution.

of wet uncoated (a) isolated microspheres showed a spherical shape. One-stage coated microspheres showed a similar structure (b) while macroscopic clumping observed during coating of C2I microspheres was confirmed by microscopic observation as can see through the existence of clustered alginate microspheres surrounded by chitosan coacervates (c).

Fig. 2a-c depicts the micrographs of individual microspheres of calcium-alginate, one-stage coated and two-stage coated microspheres, respectively. Spherical microspheres sizing up to $60 \mu \mathrm{m}$ with a smooth external surface were observed. A microspheres surface scan at high magnification caused microspheres destruction not allowing the observation of microcores.

Microsphere diameter ranged from 20 to $250 \mu \mathrm{m}$ as seen in Fig. 3. A unimodal distribution with a peak in the range of $20 \mu \mathrm{m}$ for uncoated microspheres was obtained. A broadersize distribution was seen at both coated microspheres. Onestage coated microspheres showed higher mean diameters compared to two-stage coated microspheres. Macroscopic clumping of two-stage coated microspheres, observed during either coating process and granulometric analysis, resulted in a higher mean diameter.
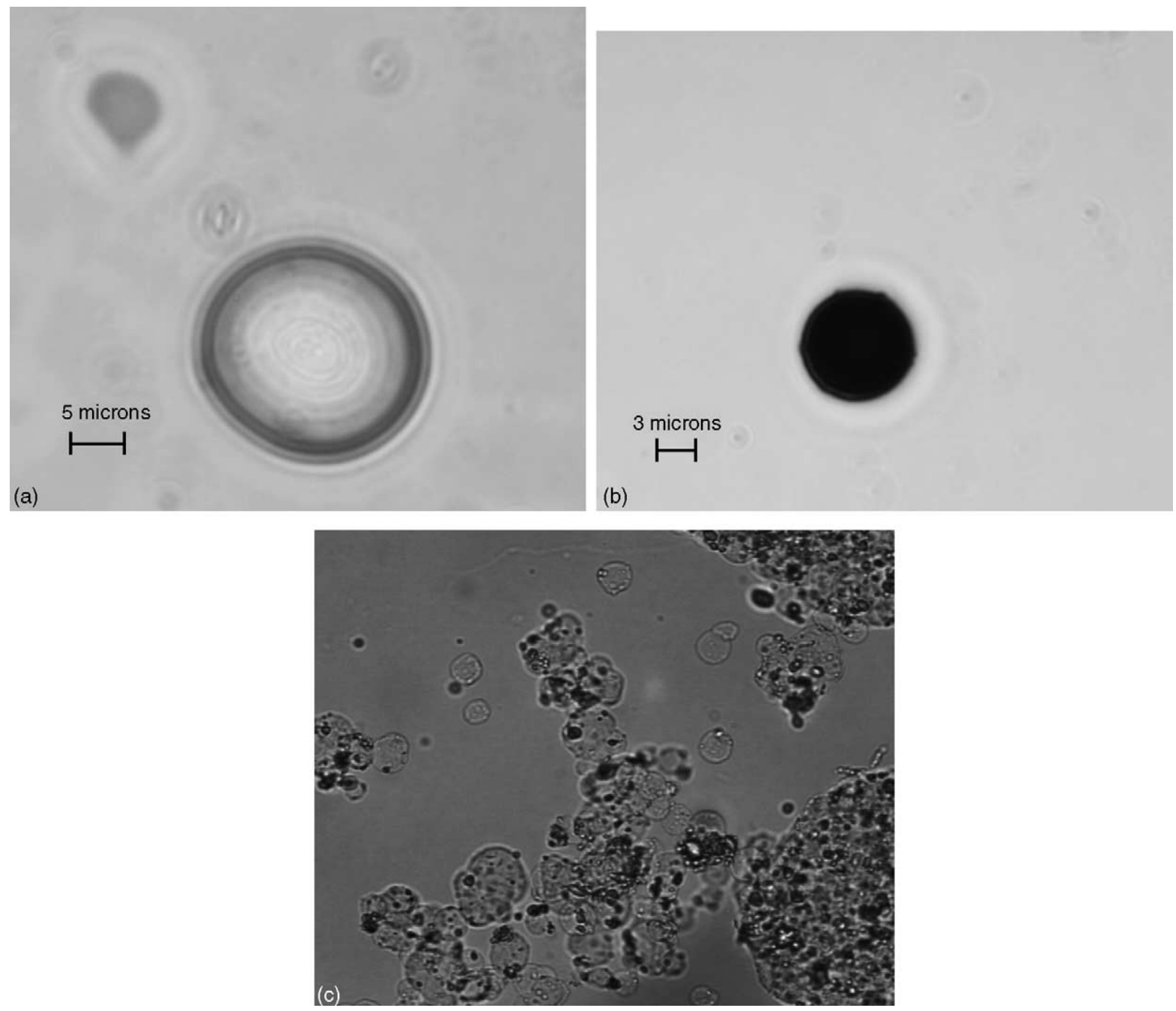

Fig. 1. Optical micrographs of recovered wet UI (a), C1I (b), and C2I (c) microspheres. 

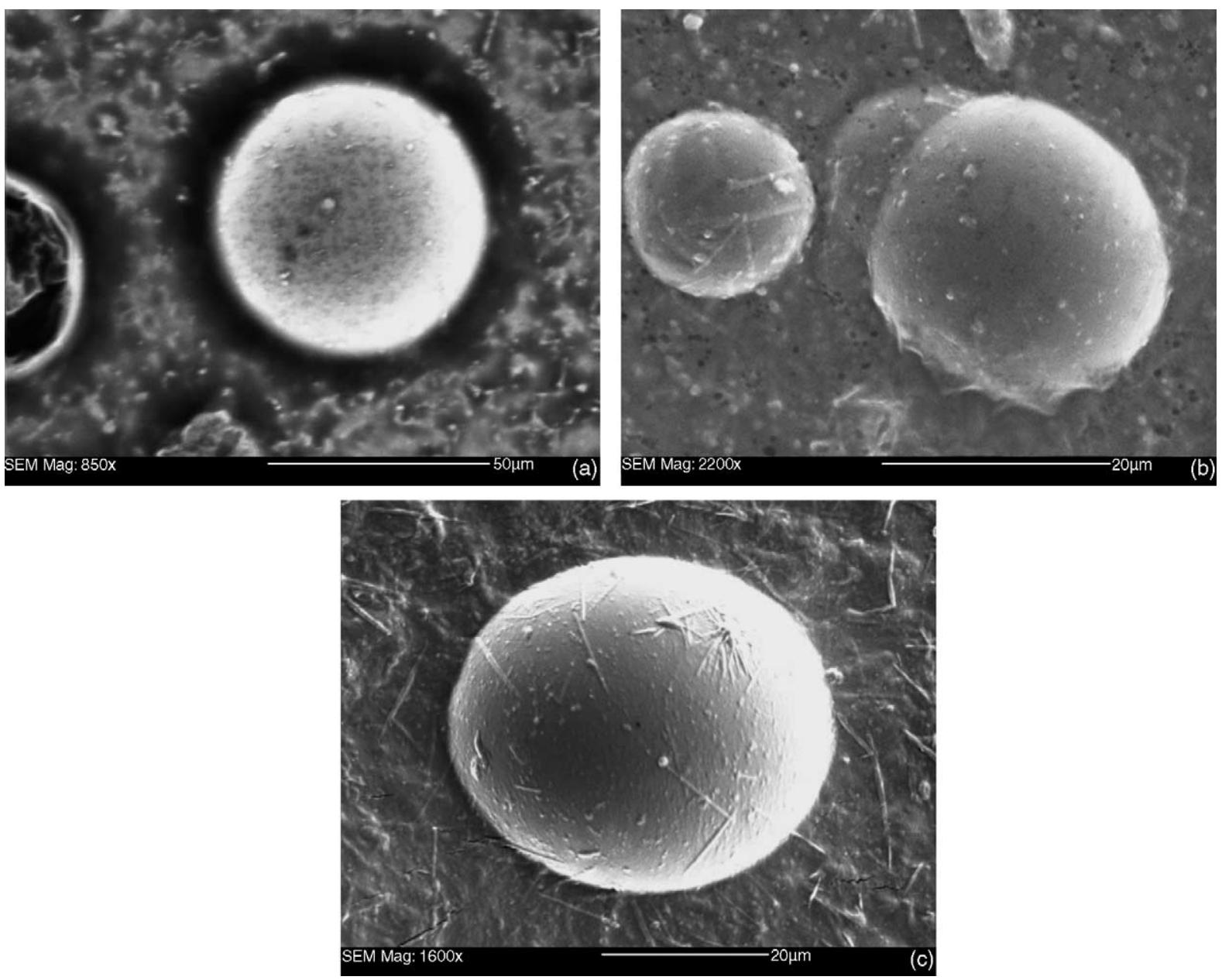

Fig. 2. Scanning electron micrographs of air-dried UI (a), C1I (b) and C2I (c) microspheres.

The DSC curves showed a broad endothermic peak between 25 and $100^{\circ} \mathrm{C}$ (Fig. 4) for isolated polyelectrolytes and its physical mixture. Chitosan-alginate complexes presented a double endothermic peak between 50 and $100^{\circ} \mathrm{C}$. All sam-

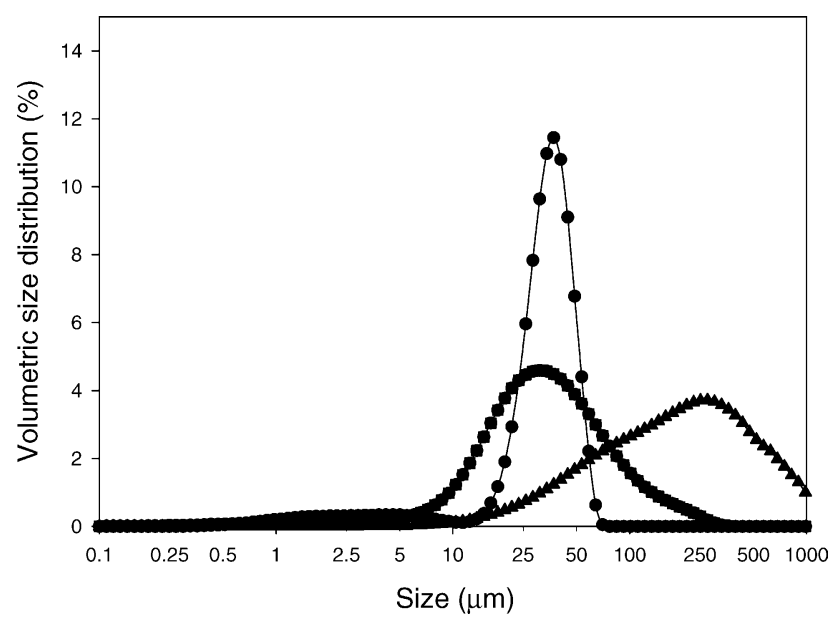

Fig. 3. Size distribution of uncoated $(\mathbf{\bullet})$, one-stage ( $\mathbf{\Lambda})$ and two-stage ( coated microspheres prepared with alginate solution in phosphate buffer at pH 6.8 and recovered with acetate buffer at $\mathrm{pH} 5.5$.

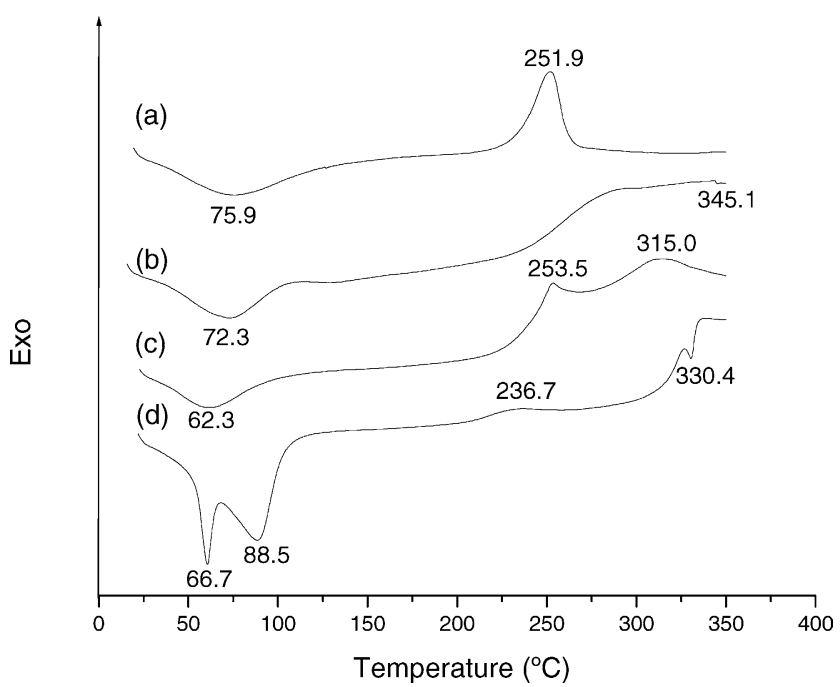

Fig. 4. Thermograms of (a) sodium alginate, (b) chitosan acetate at $\mathrm{pH} 3.5$, (c) chitosan-alginate (CS-ALG) physical mixture and (d) CS-ALG complex obtained from solutions at $\mathrm{pH}$ 5.6/4.5. 


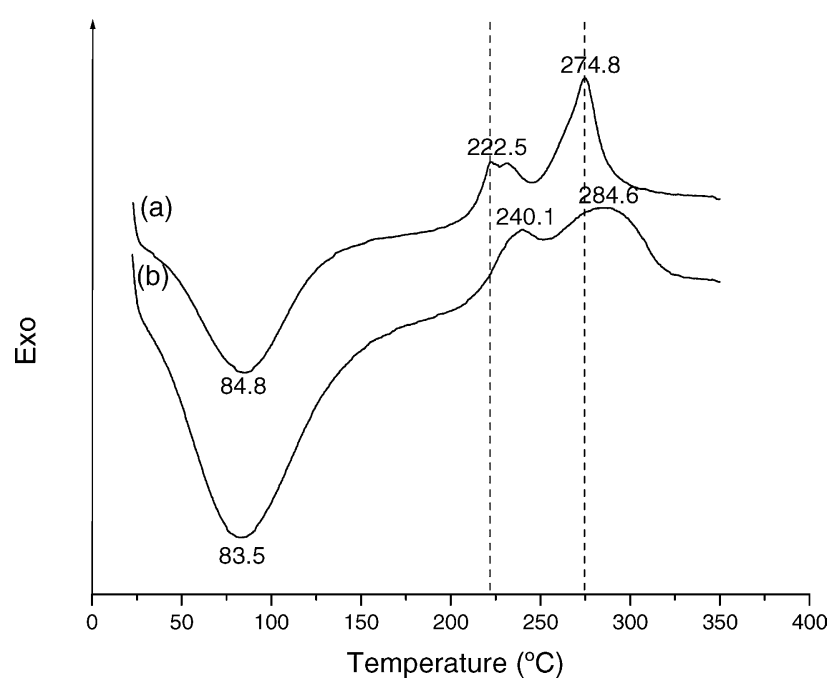

Fig. 5. Thermograms of calcium-alginate microspheres prepared by external gelation: (a) UEL and (b) CEL.

ples showed exothermic peaks between 236 and $345^{\circ} \mathrm{C}$. First exothermic peak in alginate and it physical mixture with chitosan, around $252-253^{\circ} \mathrm{C}$, was shifted to $237^{\circ} \mathrm{C}$ in CS-ALG complex.

Uncoated (UEL) and coated-alginate microspheres (CEL) prepared by external gelation presented a single endothermic peak between 25 and $125^{\circ} \mathrm{C}$ (Fig. 5) and a double exothermic peak: the first peak, similarly to CS-ALG complex, was shifted to 223 and $240{ }^{\circ} \mathrm{C}$ respectively; the second peak was observed at 275 and $285^{\circ} \mathrm{C}$ respectively.

The thermogram of internal-made microspheres exhibited either a single or a double endothermic peak. Uncoated and C1IL microspheres showed an endothermic region between 25 and $100^{\circ} \mathrm{C}$ with a peak at 67 and $70^{\circ} \mathrm{C}$, respectively, while for C2IL microspheres a double peak at 57 and $73^{\circ} \mathrm{C}$ was observed (Fig. 6). A shifting effect was detected for this double endothermic peak when comparing to the double en-

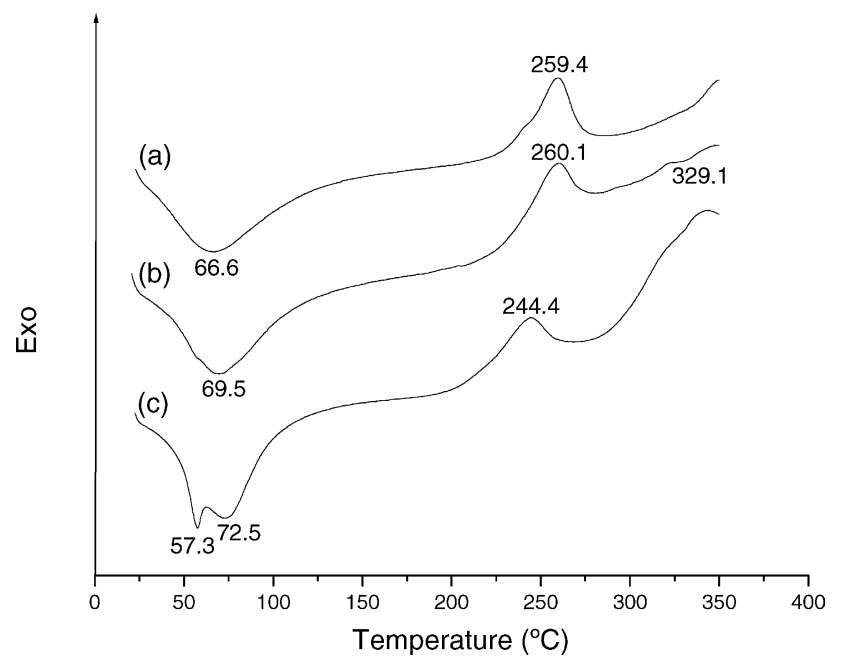

Fig. 6. Thermograms of calcium-alginate microspheres prepared by internal gelation: (a) UIL, (b) C1IL and (c) C2IL.

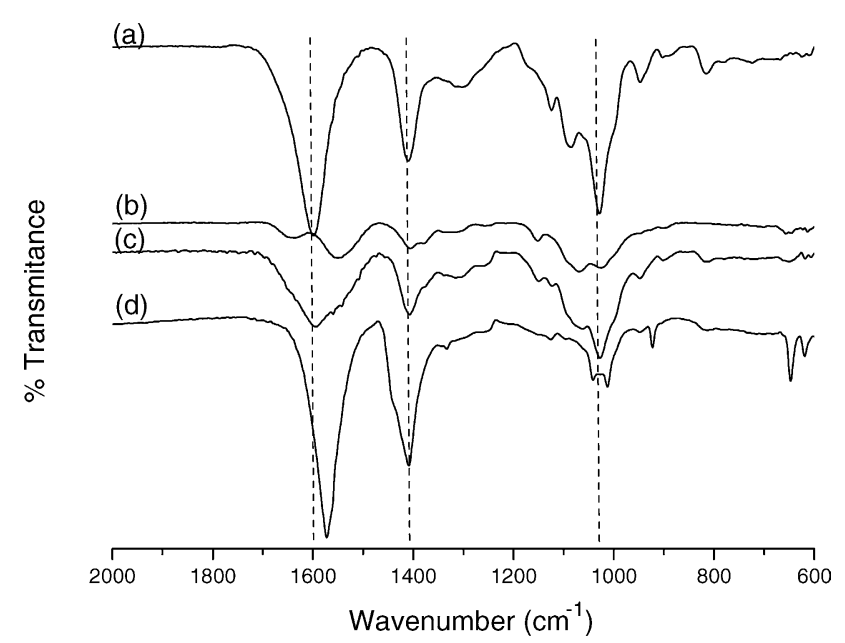

Fig. 7. FT-IR spectra of (a) sodium alginate, (b) chitosan acetate at $\mathrm{pH} 3.5$, (c) chitosan-alginate (CS-ALG) physical mixture and (d) CS-ALG complex obtained from solutions at $\mathrm{pH}$ 6.4/4.5.

dothermic observed in CS-ALG complex thermogram. An exothermic peak, around $260^{\circ} \mathrm{C}$, was observed for both UIL and C1IL, whereas C2IL showed a double exothermic peak, the first at $244^{\circ} \mathrm{C}$ and the second at $329^{\circ} \mathrm{C}$.

In order to confirm alginate-chitosan interactions, samples were analyzed by IR spectroscopy. Fig. 7 shows spectra of alginate (a), chitosan (b), physical mixture alginate-chitosan (c), and alginate-chitosan complex (d). Alginate-containing samples showed a broad band with dual peak at 1594-1602 and 1406-1410 $\mathrm{cm}^{-1}$. Chitosan spectrum showed a strong peak of amine group at $1559-1560 \mathrm{~cm}^{-1}$ and a small peak at $1641-1651 \mathrm{~cm}^{-1}$. A new peak at $1573 \mathrm{~cm}^{-1}$ was assigned to the alginate carboxylic groups for CS-ALG complex.

The FT-IR spectra of external gelation-made microspheres (Fig. 8) did not show significant differences between microspheres and alginate. Around $1000 \mathrm{~cm}^{-1}$ microspheres

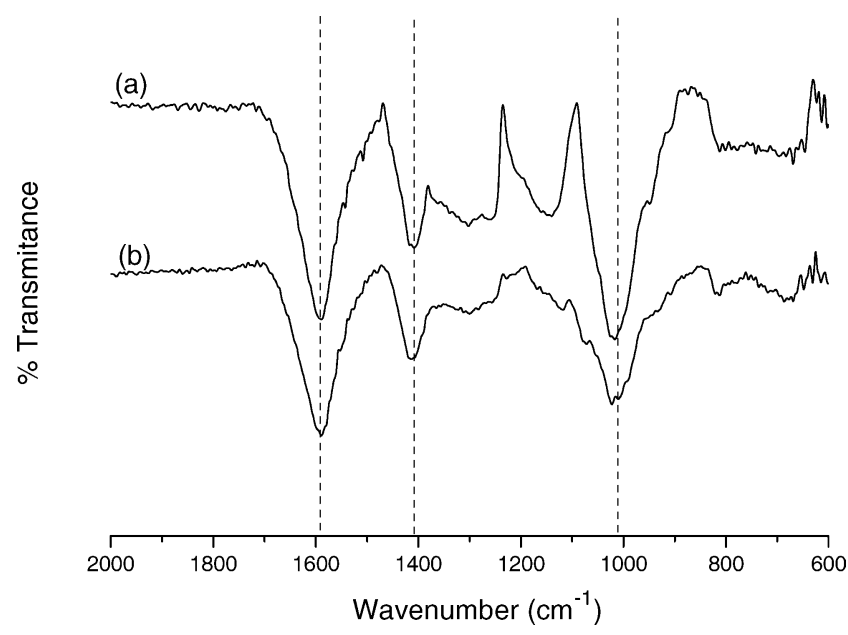

Fig. 8. FT-IR spectra of calcium-alginate microspheres prepared by external gelation: (a) UEL and (b) CEL. 


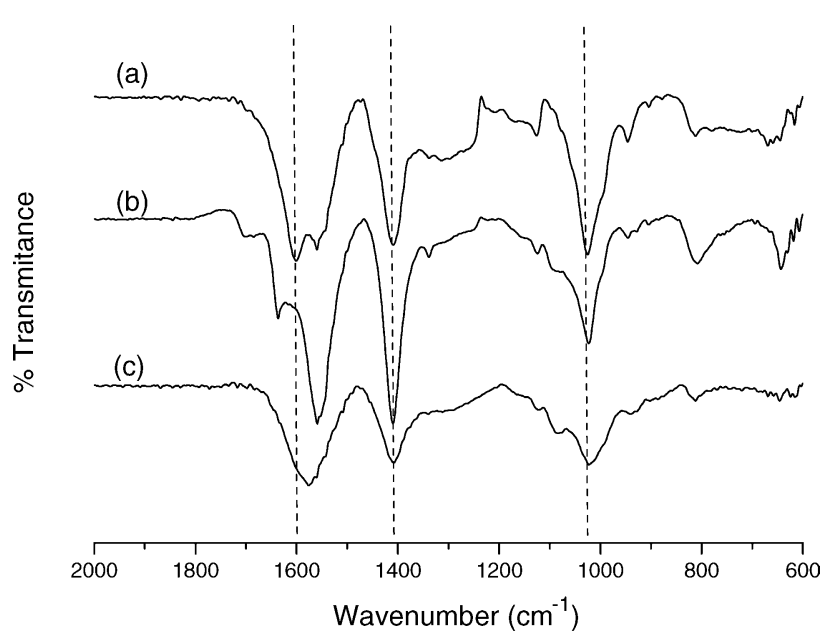

Fig. 9. FT-IR spectra of calcium-alginate microspheres prepared by internal gelation: (a) UIL, (b) C1IL, and (c) C2IL.

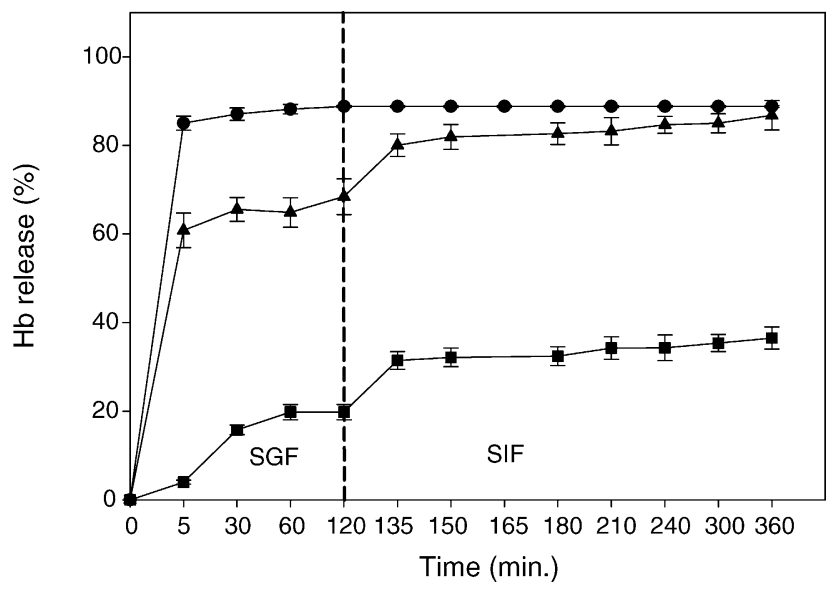

Fig. 10. In vitro release profile of $\mathrm{Hb}$ in simulated gastric fluid (SGF) for $2 \mathrm{~h}$ and then in simulated intestinal fluid (SIF) for uncoated $(\boldsymbol{O})$ one-stage $(\boldsymbol{\Lambda})$ and two-stage ( $\mathbf{\square})$ chitosan-coated alginate microspheres. All microspheres were recovered by using acetate buffer and utilized in the un-dried state. Error bars represents standard deviations about the mean based on three replicates.

showed a dual-peak similar to CS-ALG complex, more evident in coated-microspheres.

The FT-IR spectra of microspheres prepared by internal gelation showed significant differences around the region of $1400-1700 \mathrm{~cm}^{-1}$ (Fig. 9). Uncoated microspheres presented a peak around $1600 \mathrm{~cm}^{-1}$ while in $\mathrm{C} 1$ and $\mathrm{C} 2$ microspheres spectra this peak was shifted toward a lower wavenumber, $1570-1580 \mathrm{~cm}^{-1}$. Uncoated microspheres spectrum exhibited a shoulder peak at $1570 \mathrm{~cm}^{-1}$ while for $\mathrm{C} 1$ microspheres a small peak at $1640 \mathrm{~cm}^{-1}$ was assigned.

It can be seen from Fig. 10 that $\mathrm{Hb}$ release profile from microspheres in simulated gastric fluid (SGF) showed different patterns among microspheres while in intestinal fluid (SIF) a similar behavior was observed at both coated microspheres.

A fast and complete $\mathrm{Hb}$ release from uncoated microspheres, reaching $88 \%$, was observed under simulated gastric fluid (SGF). In uncoated and one-stage coated microspheres,
Table 2

$\mathrm{Hb}$ release from uncoated (UI), one-stage (C1I) and two-stage (C2I) coated microspheres after $2 \mathrm{~h}$ in simulated gastric fluid

\begin{tabular}{lll}
\hline Microspheres & Gastric release $(\%)$ & $\begin{array}{l}\text { Percentage of total release } \\
\text { (gastric release/total release) }\end{array}$ \\
\hline UI & $88.0 \pm 1.6$ & 100 \\
C1I & $68.4 \pm 4.0$ & 77.5 \\
C2I & $20.4 \pm 2.0$ & 55.5 \\
\hline
\end{tabular}

$\mathrm{Hb}$ gastric release was compared to total release.

release was dominated by a large initial burst effect. Hb released from two-stage coated microspheres was slower and its cumulative amount of $\mathrm{Hb}$ released, $20 \%$, was lower comparing to uncoated microspheres and C1I (Table 2).

In simulated intestinal fluid, a similar release rate was observed at both coated microspheres but a higher level of release, approaching $85 \%$, could be achieved from onestage coated microspheres. After a 4-h incubation of coatedmicrospheres, dark-pointed sponge-like precipitates could be seen in release media.

\section{Discussion}

The necessary conditions for successful encapsulation of $\mathrm{Hb}$ into chitosan-reinforced alginate microspheres by emulsification/internal gelation, with high encapsulation efficiency have been established.

A previous study comparing the encapsulation efficiency of bovine serum albumin (BSA) in alginate microspheres showed that internal gelation of proteins may result in a reduced encapsulation efficiency when compared to external gelation due to important losses during formulation (Vandenberg and Noue, 2001). Microspheres formed via internal gelation are more homogeneous but more porous which lead to higher DNA losses during manufacturing of DNA microspheres (Quong et al., 1998). Macromolecular interactions between negatively alginate and positively $\mathrm{Hb}$ were used to increase protein retention in the alginate matrices during production and microspheres isolation. Specifically, given hemoglobin's isoelectric point of approximately 7.1 and hence a net positive charge at $\mathrm{pH}$ below 6.0, its complexes with alginate were explored. The goals were to avoid $\mathrm{Hb}$ loss during emulsification by stabilizing the complex and during recovery steps by creating the appropriate environment, namely the $\mathrm{pH}$ and ionic force of washing medium. $\mathrm{Hb}$ losses are lower than those reported for the encapsulation of BSA and phytase, $95 \%$ and $86 \%$, respectively (Vandenberg and Noue, 2001), and DNA, 20\% (Quong et al., 1998). The high encapsulation efficiency of $\mathrm{Hb}$ is consistent with the view that a stronger protein-polyanion complex (due to enhanced either electrostatic or hydrophobic interactions), can retain protein within the alginate matrix (Kamiya and Klibanov, 2003). Studies about the effect of the structure of encapsulated materials on their release from chitosan-coated alginate microspheres revealed that $\mathrm{Hb}$, presented a lower diffusion 
compared to BSA (similar molecular weights) during formulation (Huguet and Dellacherie, 1996). Thus, other parameters such as protein degree of ionization and specific conformation can affect its encapsulation efficiency. The environmental $\mathrm{pH}$ provided by the acetate buffer, in the $\mathrm{p} K_{\mathrm{a}}$ interval of the chitosan and the alginate, has kept its global charge densities (Berger et al., 2004). Higher Hb losses in first wash of microspheres may be explained by an incomplete recovery of microspheres during phase partitioning and by the agitation destabilizing-effect during emulsification in both microspheres-networks.

Optical microscopic observation of microspheres confirms aggregation phenomena during chitosan-coating which can be explained by strong electrostatic interaction between alginate and chitosan, two polyelectrolytes of opposite charge. In the case of one-stage microspheres, coating macroscopic clumping is partially avoided by the presence of the external oil phase. The lower mean size of C2I microspheres may be explained by a lower exposition of cured alginate microspheres to chitosan when comparing to the biphasic-dispersed gelled-microspheres during the uninterrupted method. Thus, a stronger but and more uniform coating, occurring mainly at the microspheres surface, allowed the obtention of lower size two-stage coated-microspheres.

The spherical shape of microspheres may be attributed to a high degree of cross-linking occurring in each case. Externalmade alginate microspheres morphology is frequently related to a disc-like geometry due to its heterogeneous cross-linked structure, which is higher at the outer surface. The collapse of microspheres at high magnification may be attributed to a rapid and extensive dehydration upon high-energy incidence.

DSC and FT-IR analysis of the pure polysaccharides, physical mixtures thereof and microspheres were used to confirm the formation of CS-ALG complex through an uninterrupted method.

Early endotherm peaking in the temperature range $25-125^{\circ} \mathrm{C}$ related to transitions associated with loss of water correspond to the hydrophilic nature of alginate and chitosan (Zohuriaan and Shokrolahi, 2004). Furthermore, microspheres granulometry affected this transition to some extent. Higher-sizing external-made microspheres contain high moisture content, which lead to a significant shift of this endotherm peak up to $85^{\circ} \mathrm{C}$. The peak broadening also indicates that the ordered association of the alginate molecules could decrease by the presence of chitosan (Kim et al., 2002) but endothermic peak at lower temperature of its physical mixtures of polysaccharides did not confirm this possibility. Thermal behavior of polysaccharides is related to its structure and hydrophilic properties, which are affected by water activity. A reduced water activity was observed in lower sizing granules of starch (Kruger et al., 2003) and methyl cellulose (Zohuriaan and Shokrolahi, 2004). Double endothermic peaks in CS-ALG complex and C12L microspheres could be attributed to a strong interaction between the polyelectrolytes which involved a great number of alginate carboxylates and either calcium or chitosan aminogroups. Single- peaking in C1ILL microspheres and CEL microspheres may be explained by a lower cross-linking density of alginate network by chitosan due to a lower efficiency of chitosan coating. A weaker chitosan-alginate interaction in CEL microspheres could also be attributed to its higher density of calcium ions at external surface decreasing carboxylates availability and also to higher dehydration phenomena which overwhelmed single thermal behaviors. Over $200^{\circ} \mathrm{C}$, a different behavior was observed among CS-ALG complex and microspheres, but decomposition of chitosan and alginate starts at 225 and $293^{\circ} \mathrm{C}$, respectively (Zohuriaan and Shokrolahi, 2004).

FT-IR spectra showed a strong absorption around $1594-1602 \mathrm{~cm}^{-1}$ that belongs to the asymmetric stretching vibration of $-\mathrm{COO}^{-}$, and the band around $1402 \mathrm{~cm}^{-1}$ of $-\mathrm{COO}^{-}$symmetric stretching vibration (Yao et al., 1997). The carboxylate carbonyl of alginate could be shifted by the presence of calcium to $1560 \mathrm{~cm}^{-1}$ as verified with microspheres prepared by internal gelation. In the presence of chitosan, as verified with CS-ALG complex, the deviation occurred towards $1573 \mathrm{~cm}^{-1}$ due to interaction with the $-\mathrm{NH}_{3}{ }^{+}$groups of chitosan. A similar deviation was found for C1IL microspheres, suggesting the formation of a chitosan-alginate membrane during the one-stage coating process. Unlike uncoated microspheres obtained by external gelation, C1IL microspheres spectrum showed a shoulder peak at $1570 \mathrm{~cm}^{-1}$, which can be explained by a higher density of calcium-carboxylate cross-linking in microspheres obtained by internal gelation.

In C1IL microspheres the peak of $1640 \mathrm{~cm}^{-1}$ can be attributed to unreacted chitosan because surface contact between biphasic dispersed alginate microspheres and chitosan coating was not as effective as in C2I microspheres. Thus, this peak was absent in C2IL and CS-ALG complex and was attributed to amide bond of unreacted chitosan which was obtained from partial $N$-deacetylation of chitin.

The observed $\mathrm{pH}$ dependent release of $\mathrm{Hb}$ from chitosancoated alginate microspheres has been previously reported for insulin (Onal and Zihnioglu, 2002), albumin (Anal et al., 2003) and hirudin (Chandy et al., 2002). There is a lack of knowledge of alginate microspheres behaviour under simulated gastric and intestinal fluids but the stability of chitosan-alginate complex may be influenced by environmental parameters, such as $\mathrm{pH}$ and ionic strength. Two-hour assay under simulated gastric conditions can lead to dissociation of ionic linkages and dissolution of the alginate matrix (Shu et al., 2001). Alginate at the surface has a very low content of negative charges and cannot interact strongly either with calcium and positively charged chitosan (Huguet et al., 1996) and $\mathrm{Hb}$, a water-soluble macromolecule, was rapidly and completely release from destabilized uncoated alginate microspheres. We hypothesized that an uninterrupted coating method would allow sustaining $\mathrm{Hb}$ release under GI conditions but the low protein retention, especially in C1IL, suggested either a thin or a high porous chitosan-alginate membrane not acting as barrier diffusion. The weaker outer coating membrane obtained for the $\mathrm{C} 1 \mathrm{I}$ microspheres, is soluble in 

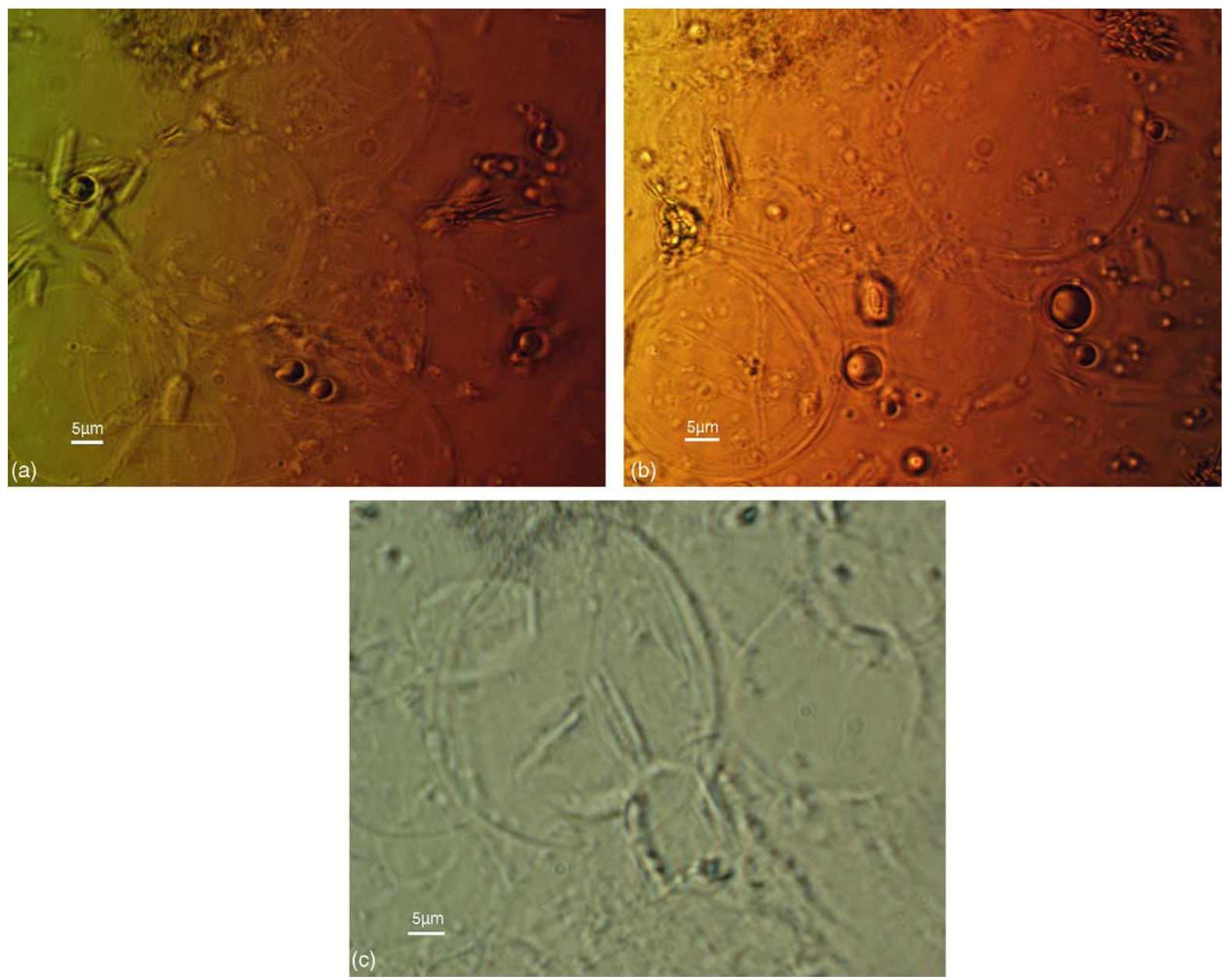

Fig. 11. Optical micrographs of C2I (a), C1I (b) after phosphate buffer release assay, and following alginate dissolution of C1I by incubation with sodium citrate $0.05 \mathrm{M}$ (c).

sufficiently acidic conditions and the barrier property of the chitosan membrane may have been lost due to its dissolution (Vachoud et al., 2001). We hypothesized that chitosan membrane could have been completed dissolved not protecting $\mathrm{Hb}$ in microspheres and to confirm the presence of chitosan membrane in release media, samples taken either after release assay and following complete alginate dissolution from coated microspheres by using sodium citrate $0.05 \mathrm{M}$. As we can see the presence of chitosan membrane in both C1I and C2I confirms that, the lower acid protection was not due to its dissolution (Fig. 11). Its microscopic features such as spherical shape size, lower than $50 \mu \mathrm{m}$, was very similar to the remained $\mathrm{C} 1 \mathrm{I}$ thin outer layer after alginate dissolution.

Since the chitosan membrane remained as a structure, at least partially, during release assay, still the hypothesis that chitosan membrane showed a high porosity, especially which concerned C2I microspheres. At pH $1.2 \mathrm{Hb}$ dissociates into dimmers $\left(M_{\mathrm{r}}=32,000\right)$ or monomer forms $\left(M_{\mathrm{r}}=16,000\right)$ which can diffuse more easily through a weaker alginate-chitosan membrane than the tetramer (Huguet et al., 1994). Microspheres swelling is ion-sensitive (Shu et al.,
2001) and $\mathrm{Hb}$ fast release from coated alginate matrix in phosphate buffer suggested a weak chitosan diffusion barrier.

The incomplete $\mathrm{Hb}$ release from coated microspheres suggests an interaction between the protein and the remained matrix. Microspheres remaining structure became agglomerated, and therefore adsorptive (Guo et al., 2004) and hydrophobic interactions between chitosan and $\mathrm{Hb}$ inside the network were favored. Chitosan molecules have both amino and hydroxyl groups that can be used to couple with proteins under mild conditions (Yang et al., 2001). Strong charge-charge interactions have been described between insulin and chitosan nanoparticles with a cumulative amount of protein release less than $25 \%$ even upon dissolution of the chitosan matrix (Ma et al., 2002).

In conclusion, chitosan-coated alginate microspheres prepared by emulsification/internal gelation represents a type of protein delivery system that can be prepared without a tedious process. In future, it should be possible to standardize the obtention of chitosan membrane in order to provide an efficient protection of protein in gastric medium and to control the release rate of protein from microspheres in intestinal medium. This can be achieved through the alteration of cross-linking 
conditions such as chitosan molecular weight and reaction time.

\section{Acknowledgements}

The authors wish to thank the Fundação Ciência e Tecnologia (FCT) of Portugal and the Cooperativa de Ensino Superior Politécnico e Universitário for financial support. Catarina Silva is the recipient from a scholarship from FCT, Portugal.

\section{References}

Anal, A.K., Bhopatkar, D., Tokura, S., Tamura, H., Stevens, W.F., 2003. Chitosan-alginate multilayer beads for gastric passage and controlled intestinal release of protein. Drug Dev. Ind. Pharm. 29, 713-724.

Berger, J., Reist, M., Mayer, J.M., Felt, O., Peppas, N.A., Gurny, R., 2004. Structure and interactions in covalently and ionically crosslinked chitosan hydrogels for biomedical applications. Eur. J. Pharm. Biopharm. 57, 19-34.

Chan, L.W., Heng, P.W., 2002. Effects of aldehydes and methods of crosslinking on properties of calcium-alginate microspheres prepared by emulsification. Biomaterials 23, 1319-1326.

Chandy, T., Rao, G.H., Wilson, R.F., Das, G.S., 2002. Delivery of LMW heparin via surface coated chitosan/peg-alginate microspheres prevents thrombosis. Drug Deliv. 9, 87-96.

Chinen, N., Tanihara, M., Nakagawa, M., Shinozaki, K., Yamamoto, E., Mizushima, Y., Suzuki, Y., 2003. Action of microparticles of heparin and alginate crosslinked gel when used as injectable artificial matrices to stabilize basic fibroblast growth factor and induce angiogenesis by controlling its release. J. Biomed. Mater. Res. 67A, 61-68.

Coppi, G., Iannuccelli, V., Leo, E., Bernabei, M.T., Cameroni, R., 2002. Protein immobilization in crosslinked alginate microparticles. J. Microencapsul. 19, 37-44.

Guo, T.Y., Xia, Y.Q., Hao, G.J., Song, M.D., Zhang, B.H., 2004. Adsorptive separation of hemoglobin by molecularly imprinted chitosan beads 1370. Biomaterials.

Haas, S., Miura, F., Zavala, F., Murata, K., Leone, B., Santiago, N., 1996. Oral immunization with a model protein entrapped in microspheres prepared from derivatized alpha-amino acids. Vaccine 14, 785-791.

Hoffman, A.S., 2001. Hydrogels for biomedical applications. Ann. NY Acad. Sci. 944, 62-73.

Huguet, M.L., Dellacherie, E., 1996. Calcium-alginate beads coated with chitosan: effect of the structure of encapsulated materials on their release. Proc. Biochem. 31, 745-751.

Huguet, M.L., Groboillot, A., Neufeld, R.J., Poncelet, D., Dellacherie, E., 1994. Hemoglobin encapsulation in chitosan/calcium-alginate beads. J. Appl. Polym. Sci. 51, 1427-1432.

Huguet, M., Neufeld, R., Dellacherie, E., 1996. Calcium-alginate beads coated with polyanionic polymers: comparison of chitosan and DEAEdextran. Proc. Biochem. 31, 347-353.

Kamiya, N., Klibanov, A.M., 2003. Controling the rate of protein release from polyelectrolyte complexes. Biotechnol. Bioeng. 82, 590-594.

Kierstan, M., Bucke, C., 1977. The immobilization of microbial cells, subcellular organelles, and enzymes in calcium-alginate gels. Biotechnol. Bioeng. 19, 387-397.

Kim, S.J., Park, S.J., Shin, M.S., Lee, Y.H., Kim, N.G., Kim, S.I., 2002. Thermal characteristics of IPNs composed of polyallylamine and chitosan. J. Appl. Polym. Sci. 85, 1956-1960.

Ko, C., Dixit, V., Shaw, W., Gitnick, G., 1995. In vitro slow release profile of endothelial cell growth factor immobilized within calcium-alginate microbeads. Artif. Cell. Blood Substit. Immobil. Biotechnol. 23, 143-151.

Kruger, A., Ferrero, C., Zaritzky, N.E., 2003. Modelling corn starch swelling in batch systems: effect of sucrose and hydrocolloids. J. Food Technol. 58, 125-133.

Larisch, B.C., Poncelet, D., Champagne, C.P., Neufeld, R.J., 1994. Microencapsulation of Lactococcus lactis subsp. cremoris. J. Microencapsul. 11, 189-195.

Liu, L.S., Liu, S.Q., Ng, S.Y., Froix, M., Heller, J., 1997. Controlled release of interleukin 2 for tumor immunotherapy using alginate/chitosan porous microspheres. J. Contr. Rel. 43, 65-74.

Ma, Z., Yeoh, H.H., Lim, L.Y., 2002. Formulation pH modulates the interaction of insulin with chitosan nanoparticles. J. Pharm. Sci. 91, 1396-1404.

Machluf, M., Orsola, A., Atala, A., 2000. Controlled release of therapeutic agents: slow delivery and cell encapsulation. World J. Urol. 18, 80-83.

Murata, Y., Maeda, T., Miyamoto, E., Kawashima, S., 1993. Preparation of chitosan-reinforced alginate gel beads-effects of chitosan on gel matrix erosion. Int. J. Pharm. 96, 130-145.

Onal, S., Zihnioglu, F., 2002. Encapsulation of insulin in chitosan-coated alginate beads: oral therapeutic peptide delivery. Artif. Cell. Blood Substit. Immobil. Biotechnol. 30, 229-237.

Pariente, J.L., Kim, B.S., Atala, A., 2001. In vitro biocompatibility assessment of naturally derived and synthetic biomaterials using normal human urothelial cells. J. Biomed. Mater. Res. 55, 33-39.

Poncelet, D., Poncelet de Smet, B., Beaulieu, C., Neufeld, R.J., 1992a. Scale-up of gel microcapsule and microcapsule production in cell immobilization. In: Goosen, MF. (Ed.), Fundamentals of Animal Cell Encapsulation and Immobilization. CRC Press, Boca Raton.

Poncelet, D., Lencki, R., Beaulieu, C., Halle, J.P., Neufeld, R.J., Fournier, A., 1992b. Production of alginate beads by emulsification/internal gelation. I. Methodology. Appl. Microbiol. Biotechnol. 38, 39-45.

Quong, D., Neufeld, R.J., 1998. DNA protection from extracapsular nucleases, within chitosan- or poly-L-lysine-coated alginate beads. Biotechnol. Bioeng. 60, 124-134.

Quong, D., Neufeld, R.J., Skjak, B., Poncelet, D., 1998. External versus internal source of calcium during the gelation of alginate beads for DNA encapsulation. Biotechnol. Bioeng. 57, 438-446.

Ribeiro, A.J., Neufeld, R.J., Arnaud, P., Chaumeil, J.C., 1999. Microencapsulation of lipophilic drugs in chitosan-coated alginate microspheres. Int. J. Pharm. 187, 115-123.

Shu, X.Z., Zhu, K.J., Song, W., 2001. Novel pH-sensitive citrate crosslinked chitosan film for drug controlled release. Int. J. Pharm. 212, $19-28$.

Stevens, M.M., Qanadilo, H.F., Langer, R., Prasad, S.V., 2004. A rapidcuring alginate gel system: utility in periosteum-derived cartilage tissue engineering. Biomaterials 25, 887-894.

Suzuki, Y., 1998. Determination of human hemoglobin in blood based on its spectral change due to the solvent effect of ethanol 2882. Anal. Sci. 14, 1013-1016.

Vachoud, L., Zydowicz, N., Domard, A., 2001. Sorption and desorption studies on chitin gels. Int. J. Biol. Macromol. 28, 93-101.

Vandenberg, G.W., Noue, J., 2001. Evaluation of protein release from chitosan-alginate microcapsules produced using external or internal gelation. J. Microencapsul. 18, 433-441.

Wang, K., He, Z., 2002. Alginate-konjac glucomannan-chitosan beads as controlled release matrix. Int. J. Pharm. 244, 117-126.

Yang, L., Hsiao, W.W., Cheng, P., 2001. Chitosan-cellulose composite membrane for affinity purification of biopolymers and immunoadsorption. J. Membr. Sci. 5084, 1-13.

Yao, k., Tu, h., Cheng, F., Zhang, J., Liu, J., 1997. pH-sensitivity of the swelling of a chitosan-pectin polyelecrolyte complex. Die Angew. Makromol. Chem. 245, 63-72.

Zohuriaan, M.J., Shokrolahi, F., 2004. Thermal studies on natural and modified gums 3458. Polym. Test. 23, 575-579. 